\title{
So schlecht ist TV für die Gesundheit
}

\author{
Wer seine Freizeit vor dem Fernsehgerät verbringt, \\ sammelt zweifelsohne reichlich Risikofaktoren für \\ Herz-Kreislauf-Erkrankungen. In Australien wurde \\ jetzt geprüft, ob es einen direkten Zusammenhang \\ zwischen dem Ausmaß des Fernsehkonsums und der \\ kardiovaskulären Mortalität gibt.
}

- Forscher des Diabetesforschungsinstituts Melbourne prüften innerhalb der Australian Diabetes, Obesity and Lifestyle Study (Ausdiab) über 6,6 Jahre bei 8800 über 25 Jahre alten Australiern die Beziehungen zwischen TV-Zeit und den verschiedenen Todesursachen.

Während der Beobachtungszeit verstarben insgesamt 284 Teilnehmer, davon 87 an Herz-Kreislauf-Krankheiten und 125 an Krebs. Jede während der sechseinhalb Jahre täglich vor dem Fernsehgerät verbrachte Stunde steigerte das generelle Sterblichkeitsrisiko um 11\% (HR 1,11; 95\% $\mathrm{K}=1,03-1,35$ ), das für Herz-Kreislauf-Krankheiten (HKK) um $18 \%$ (HR 1,18; 95\% KI = 1,03-1,5) und Krebs 9\% (HR 1,09). Die Gefahr, einer Herz-Kreislauf-Erkrankung zu erliegen, stieg pro zusätzlicher Stunde täglich um $18 \%$.

Die Forscher verglichen Teilnehmer, die täglich weniger als zwei Stunden (HR 1,13; 95\%-KI = 0,87-1,36) fernsahen, mit Teilnehmern, die pro Tag mehr als vier Stunden in die Röhre glotzten. Sie stellten bei den Vielsehern eine um mehr als die Hälfte erhöhte allgemeine Sterblichkeit fest (HR 1,46; 95\%-KI=1,04-2,05) und ein um 80\% höheres Risiko (HR 1,80; 95\%-KI = 1,00-3,00), an HerzKreislauf-Erkrankungen zu sterben. Das Resultat blieb auch dann bestehen, wenn die Forscher andere Einflussfaktoren wie z. B. Rauchen und Bluthochdruck beachteten.

KOMMENTAR: Die TV-Zeit ist mit der erhöhten, allgemeinen und durch Herz-Kreislauf-Krankheiten bedingten Sterblichkeit hoch assoziiert. Präventionsstrategien für chronische Krankheiten sollten demnach nicht nur körperliches Training propagieren, sondern auch darauf abzielen, dass weniger Zeit vor dem Fernsehgerät vertan wird.

K. MALBERG

- D. W. Dunstan et al.

(Baker IDI Heart and Diabetes Institute, Melbourne, Australia; e-mail: david.dunstan@bakeridi.edu.au) Television viewing time and mortality. The australian diabetes, obesity and lifestyle study (Aus Diab). Published online before print on January 11. 2010, 10.1161/CIRCULATIONAHA.109.894824 